\title{
VCSELs with a high-index-contrast grating for mode-division multiplexing
}

\author{
Ran, Qijiang; Chung, II-Sug
}

Publication date:

2013

Document Version

Publisher's PDF, also known as Version of record

Link back to DTU Orbit

Citation (APA):

Ran, Q. (Author), \& Chung, I-S. (Author). (2013). VCSELs with a high-index-contrast grating for mode-division multiplexing. Sound/Visual production (digital)

\section{General rights}

Copyright and moral rights for the publications made accessible in the public portal are retained by the authors and/or other copyright owners and it is a condition of accessing publications that users recognise and abide by the legal requirements associated with these rights.

- Users may download and print one copy of any publication from the public portal for the purpose of private study or research.

- You may not further distribute the material or use it for any profit-making activity or commercial gain

- You may freely distribute the URL identifying the publication in the public portal

If you believe that this document breaches copyright please contact us providing details, and we will remove access to the work immediately and investigate your claim 


\section{3-08}

\section{VCSEL with a high-index-contrast grating for mode-division multiplexing}

Qijiang Ran and Il-Sug Chung

DTU Fotonik

Department of Photonics Engineering

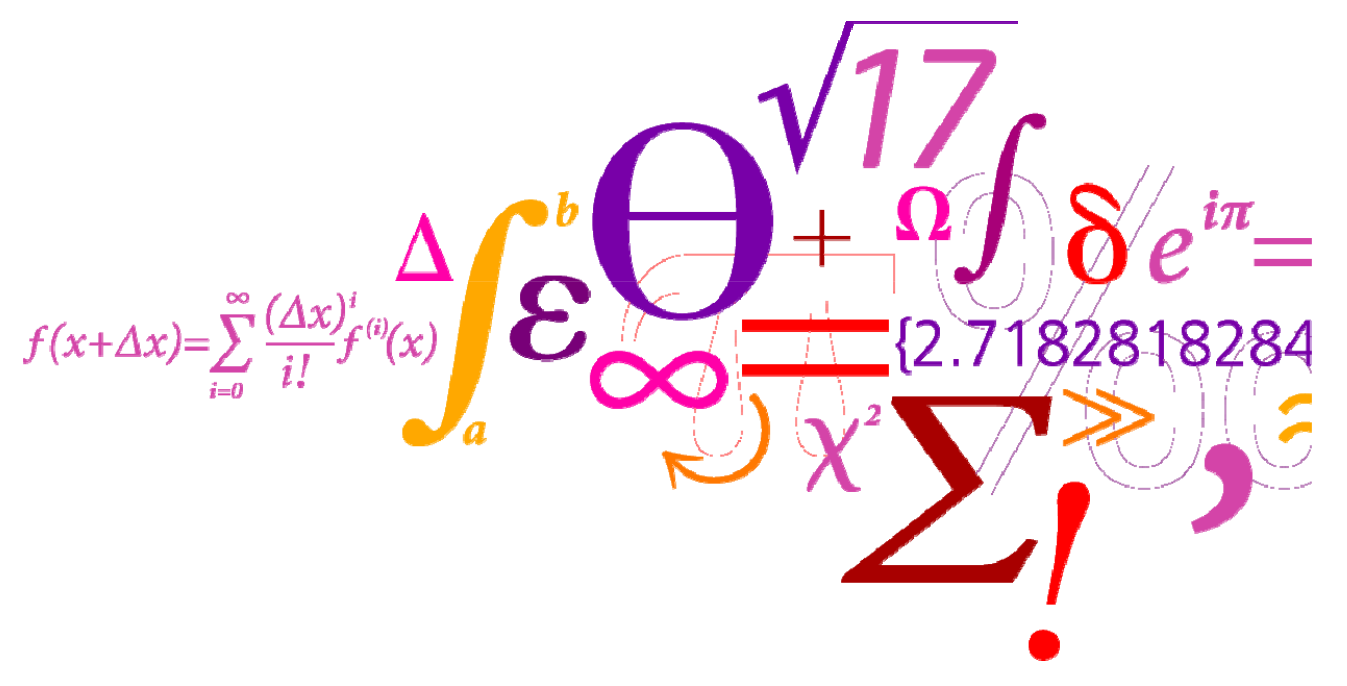




\section{Outline}

- Spatial degree of freedom for higher bandwidth

- Prior methods for exciting LP01 modes

- Proposed way: Reflectivity-modulated HCG VCSELS

- Results and discussion

- Summary 


\section{Using spatial degree of freedom}

- Space division multiplexing

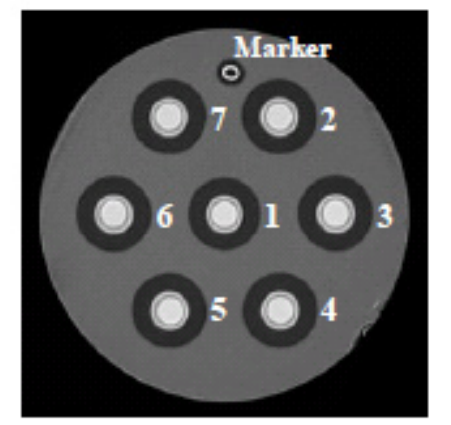

$\mathrm{LP}_{01}$ (b)

$\mathrm{LP}_{11 \mathrm{a}}$
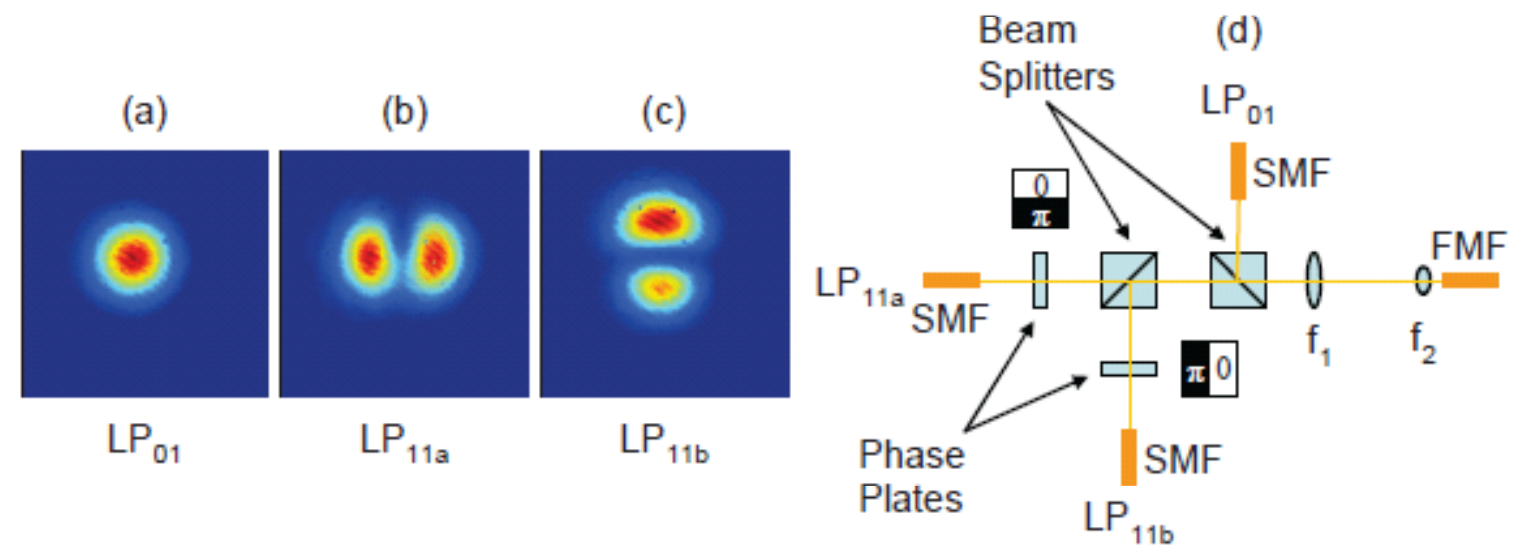

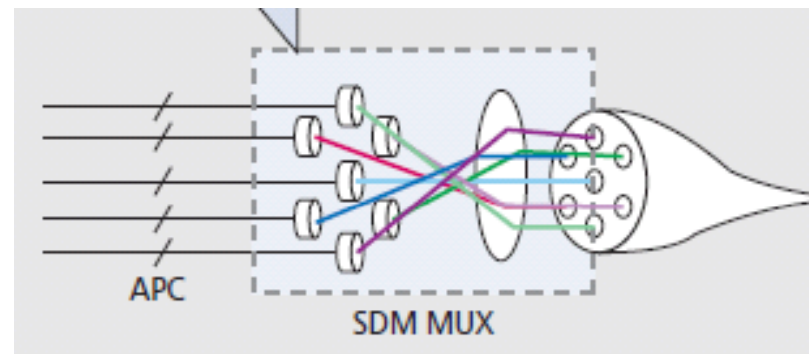

Morioka et al., IEEE ComMag S31 (Feb 2012)

Sakaguchi et al., OFC, PDPB6 (2011)

- Mode division multiplexing

Randel et al., Opt Express 19, 16697 (2011) 


\section{Exciting $\mathrm{LP}_{11}$ modes}
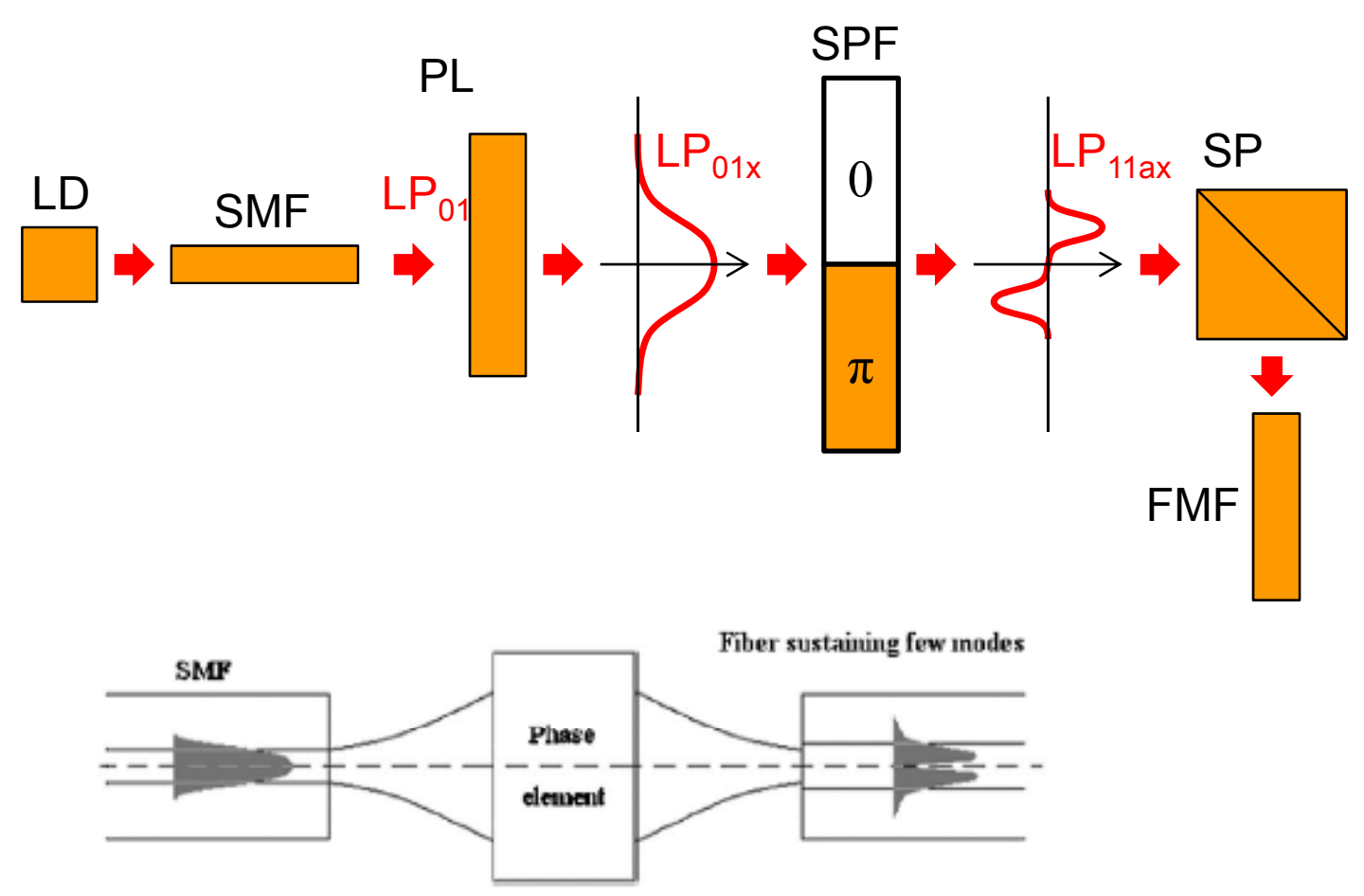

Randel et al., Opt Express 19, 16697 (2011)

Mohammed et al., Opt Eng 45, 074602 (2006) 


\section{Exciting $\mathrm{LP}_{\mathbf{1 1}}$ modes}

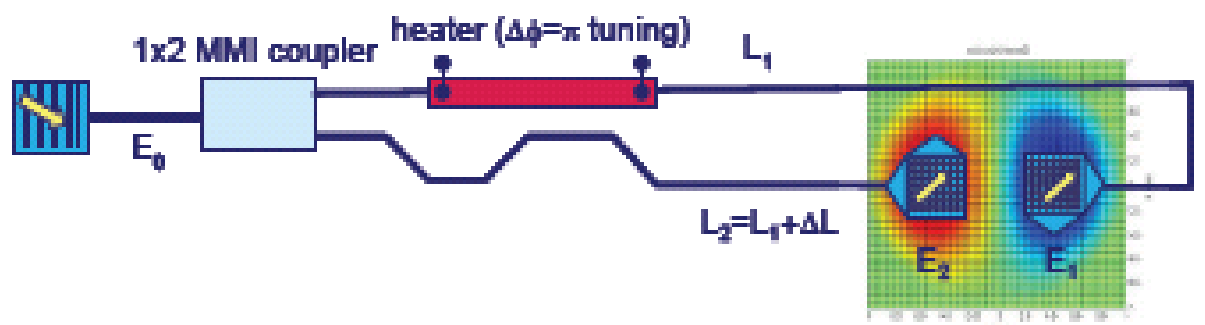

(b)

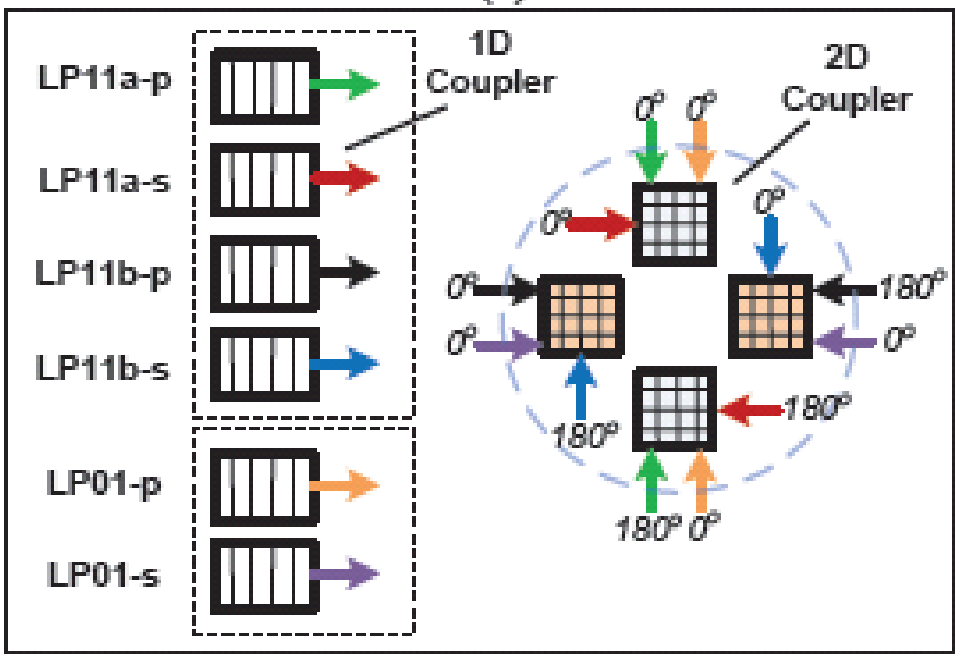

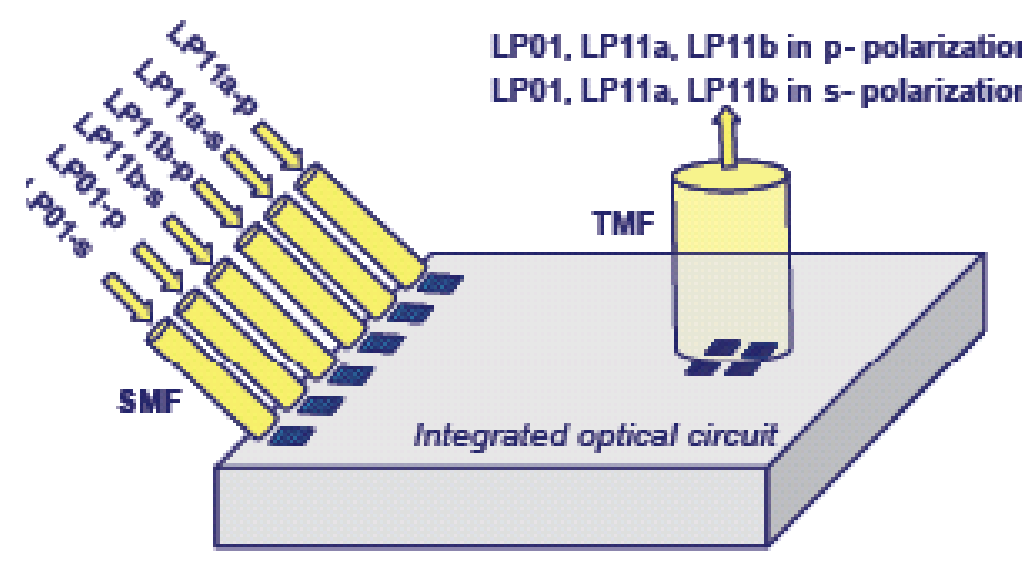

(d) 


\section{Proposed monolithic approach
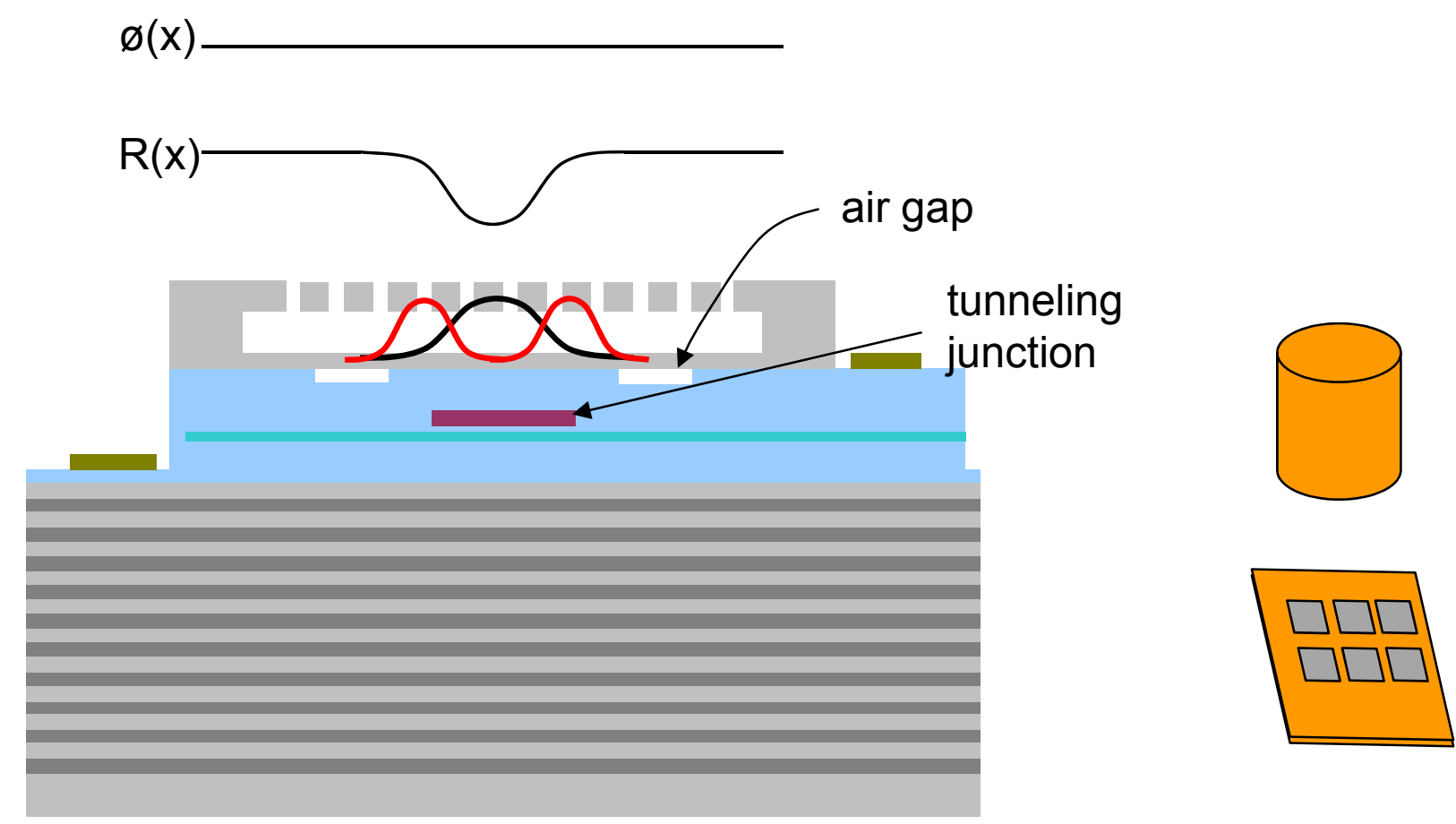


\section{Modulation of phase profile}

Engineering phase profile while keeping reflectivity profile constant.

Local phase and reflectivity is determined by local grating parameters.

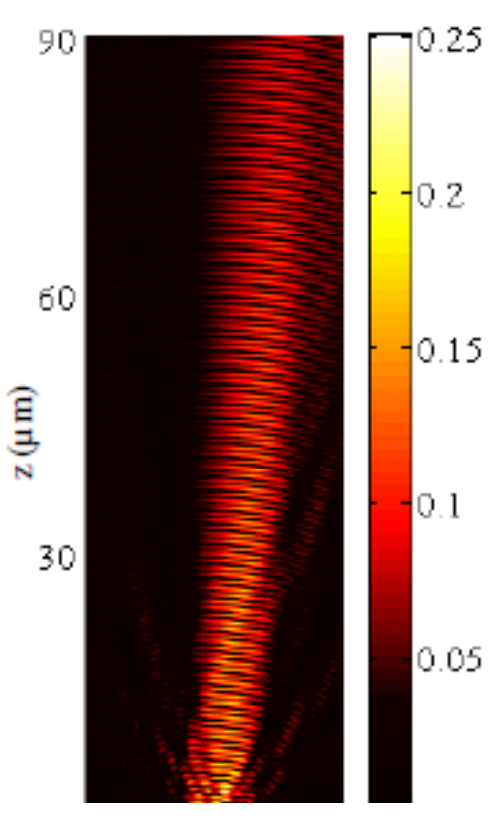

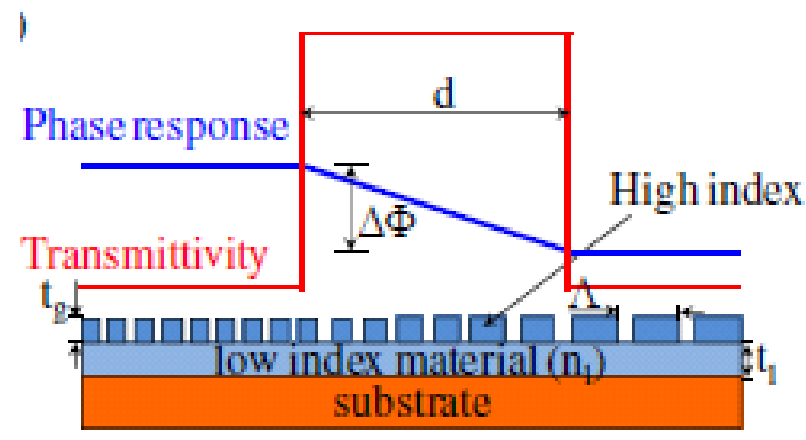

7

PW 2013, Feb 5, 2013

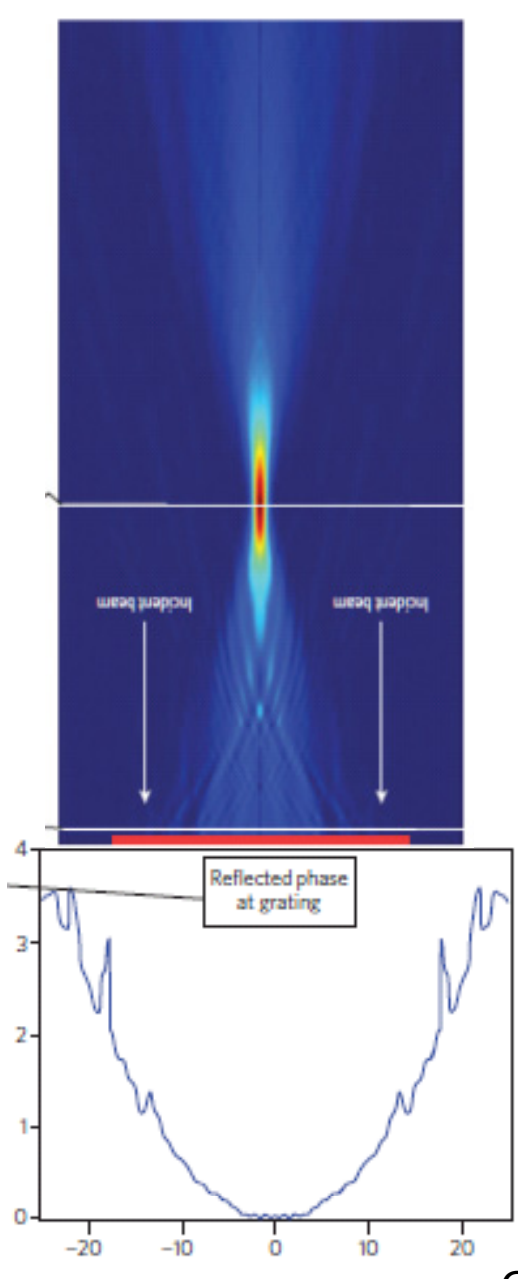

Carletti et al., Opt Express 19, 23570 (2012). Fattal et al, Nat Photon 4, 466 (2011) Lu et al, 4, 466, Nat Photon (2011) 


\section{Modulation of reflectivity profile}

Idea: Controlling local R profile while keeping phase profile.
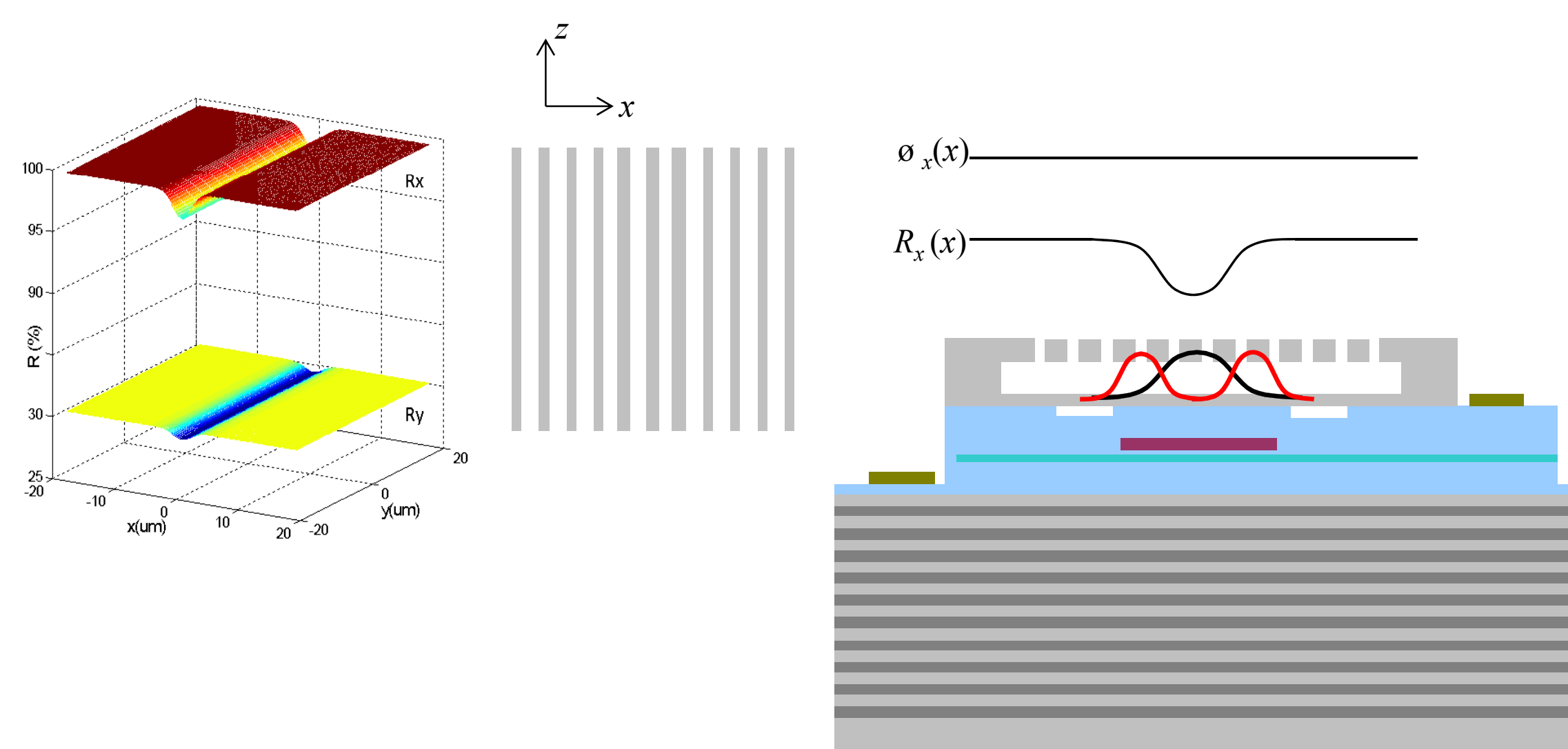


\section{Proposed way}

For LP11

$\varnothing_{x}(x)$

$R_{x}(x)$

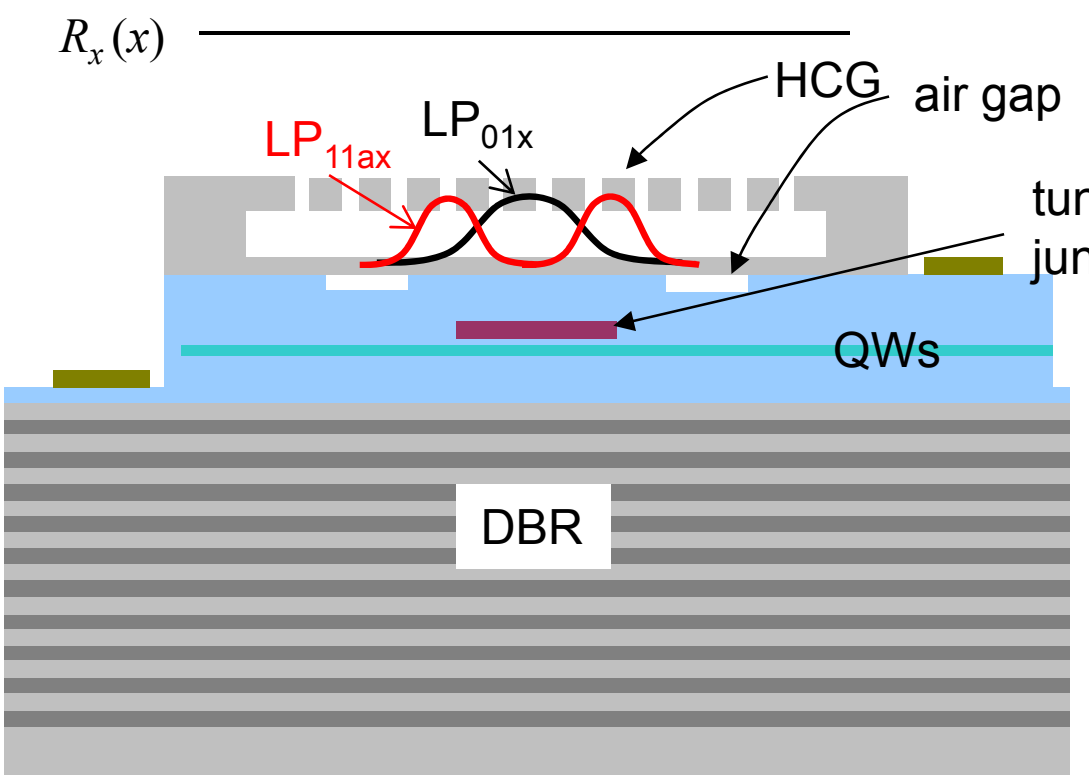

For LP01

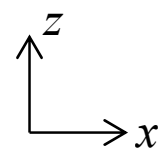

$\varnothing_{x}(x)$

$$
R_{x}(x)
$$

tunneling

junction
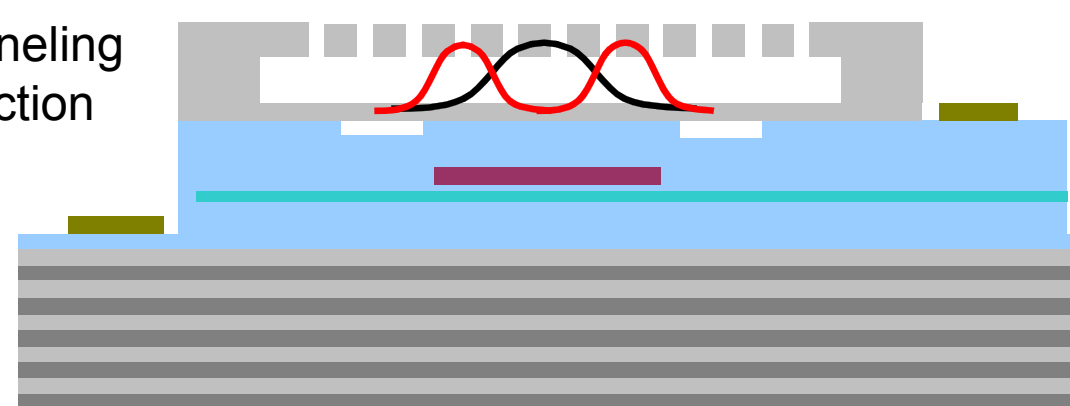


\section{Selective pumping + k-selection}

- Strongly enhanced single-mode strength

- 115\% (TJ) + 65\% (HCG) $=215 \%$ (HCG TJ)

- The HCG region is much larger than the optical modes.

- Selective pumping + inherent HCG mode selection brings synergy.
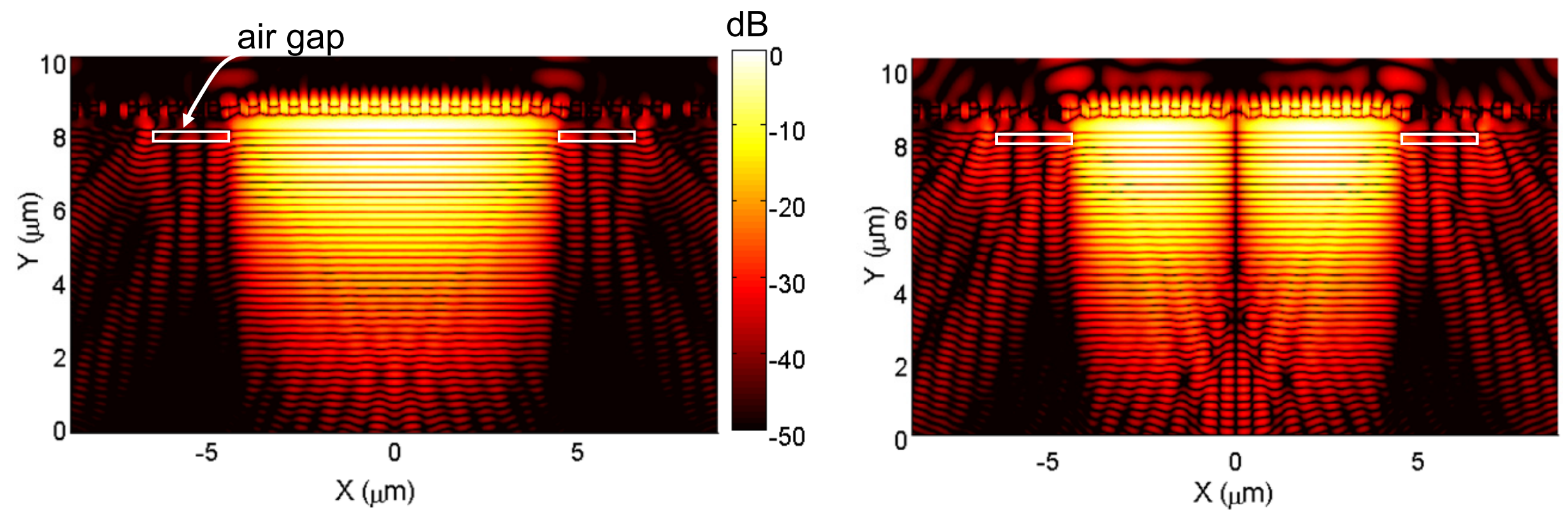


\section{Selecting LP01ax}
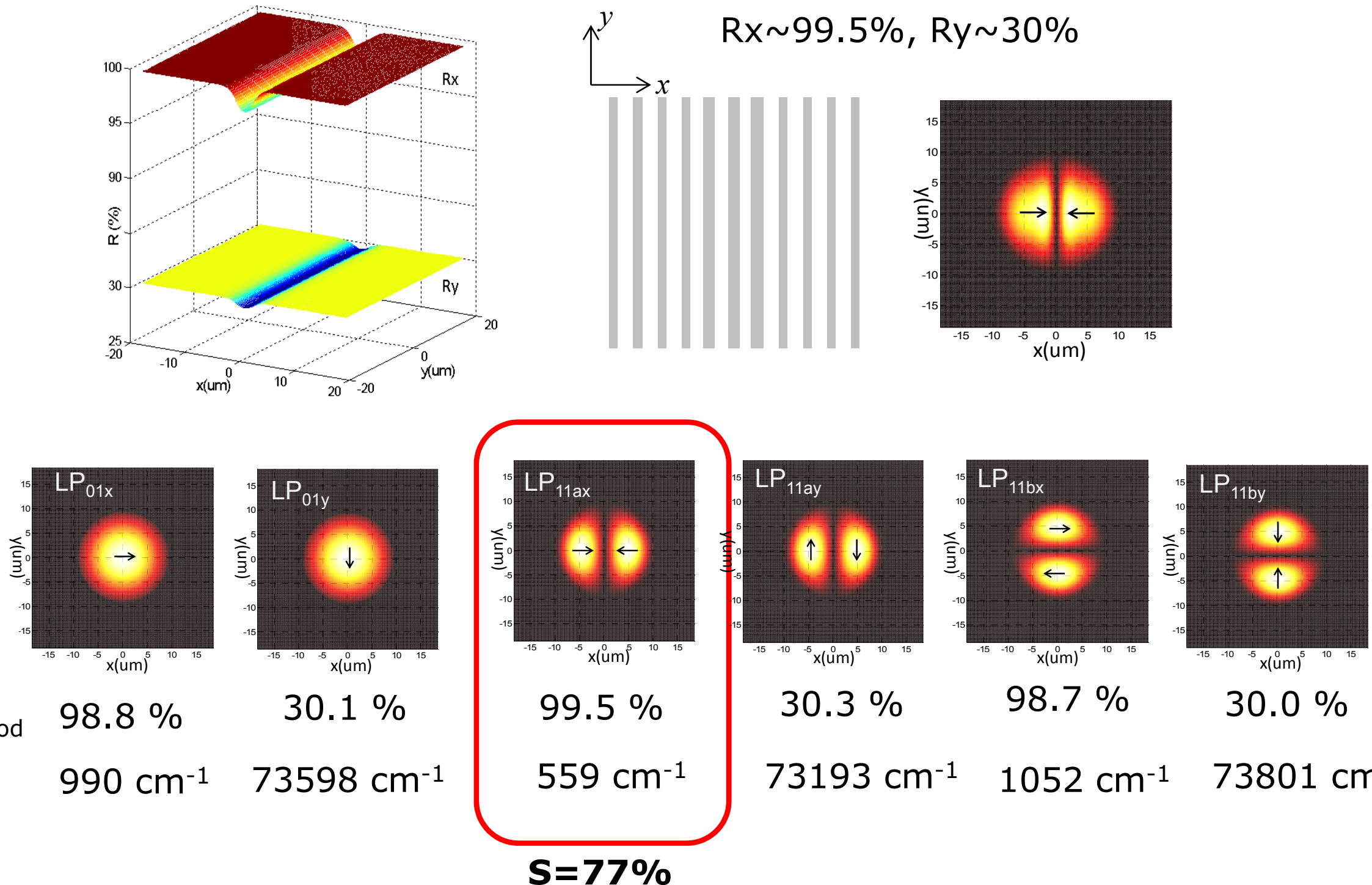

$\mathrm{R}_{\bmod } \quad 98.8 \%$

$30.1 \%$

$30.3 \%$

$98.7 \%$

$30.0 \%$

$g_{\text {th }} \quad 990 \mathrm{~cm}^{-1}$

$73598 \mathrm{~cm}^{-1}$

$S=77 \%$ 


\section{Selecting other LP01 modes}
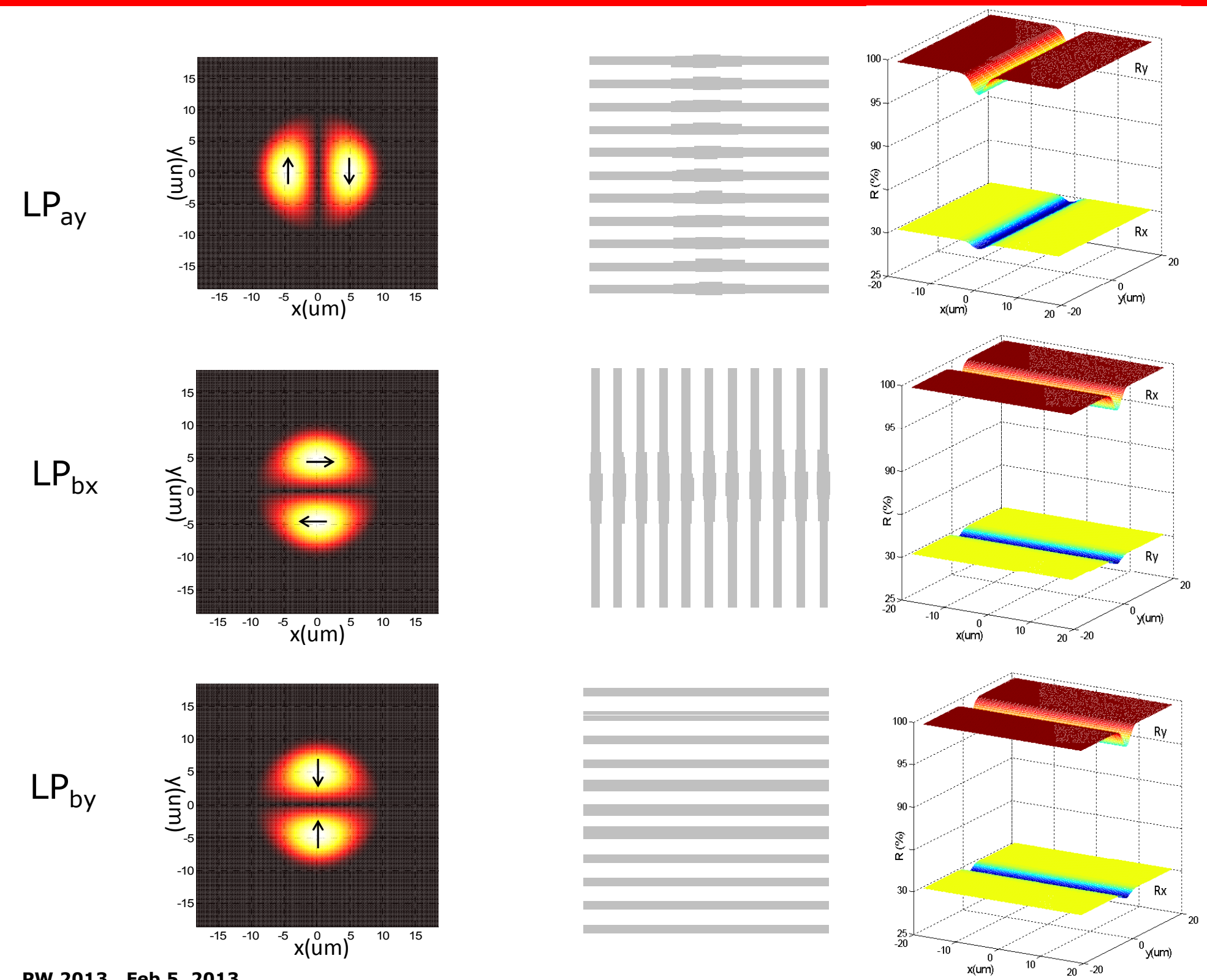


\section{Summary}

- Shaping reflectivity profile of a HCG VCSEL enables to selectively emit LP01 modes

- Moderate single mode operation for LP01 modes

- Very strong single-mode operation for LP11 modes

- Possibility of combining beam tilting 